\title{
ДЕЙСТВИТЕЛЬНО ЛИ ОЖИДАЕМАЯ \\ ПРОДОЛЖИТЕЛЬНОСТЬ ЖИЗНИ ПРИ РОЖДЕНИИ \\ ЯВЛЯЕТСЯ НАИЛУЧШИМ ИЗМЕРИТЕЛЕМ \\ УРОВНЯ СМЕРТНОСТИ НАСЕЛЕНИЯ?
}

\section{ЕВГЕНИЙ АНДРЕЕВ}

\begin{abstract}
Принято считать, что наиболее адекватной характеристикой уровня смертности населения в некоторый период времени является показатель «ожидаемая продолжстельность жизни при рождении» (ОПЖ). Данный показатель имеет серьезные недостатки, а подобный выбор создает ряд неоправданных трудностей. Главный недостаток - метод расчета ОПЖ до сих пор не унифицирован и, скорее всего, унифицирован быть не может. В силу этого ОПЖ для разных стран и периодов могут быть несравнимы. Данные международной Hитап Life-Table Database позволяют утверждать, что часто при сравнениях нельзя использовать первый десятичный знак.
\end{abstract}

Определение ОПЖ требует расчета таблиц смертности для условного поколения календарного периода. Когда уровень смертности быстро снижается, как это происходит в больиинстве стран с середины XX века, таблицы смертности условного поколения мало что говорят о возрастных закономерностях смертности когорт, но, скорее, формируют ложные впечатления. Подсчет числа человек в условном поколении, которые доживают или не доживают до некоторого возраста, не имеет отношения ни к какой реальной совокупности. Лишь расчет ОПЖ оправдывает построение таблии смертности.

Переход от возрастных показателей смертности $к$ ОПЖ описывается не формулой, а вычислительной процедурой. Это создает серьезные трудности при попытках оценить влияние на ОПЖ смертности отдельных возрастов, от отдельных причин смерти, в отдельных регионах и группах населения.

Всеми этими недостатками не обладает стандартизованный коэффициент смертности (СКС), расчет которого становится безальтернативным, как только задано стандартное население. Данные Human Mortality Database (Demographic Research 2021) позволили показать, что оченка уровня смертности с помощью СКС почти совпадает с оченкой на основе ОПЖ.

СКС - линейная функция возрастных коэффиџиентов смертности, поэтому расчет влияния смертности отдельных возрастов, причин смерти, смертности регионов или групп населения на СКС осуществить несложно.

Непрофессиональ воспринимают ОПЖ на интуитивном уровне как длительность человеческой жизни, что, скорее, недостаток показателя. Использование СКС требует больше объяснений. Но в практических исследованиях и в профессиональных публикациях ориентация на СКС облегчит работу и защитит от необоснованных эмоциий.

Ключевые слова: смертность, ожидаемая продолжительность жизни, стандартизованный коэффициент смертности, уровень смертности.

ЕВГЕНИЙ МИХАЙЛОВИч АНДРЕЕВ (e.andreev@hse.ru), НАЦИОНАЛЬНЫЙ ИССЛЕДОВАТЕЛЬСКИЙ УНИВЕРСИТЕТ «ВЫСШАЯ ШКОЛА ЭКОНОМИКИ», РОсСИЯ.

СТАТЬЯ ПОСТУПИЛА В РЕДАКЦИЮ В МАРТЕ 2021 Г. 


\section{ВВЕДЕНИЕ}

Принято считать, что наиболее адекватной характеристикой уровня смертности в населении в некоторый период времени является показатель «ожидаемая продолжительность жизни при рождении» (это длинное название современных публикациях на русском обычно заменяется на ОПЖ).

Показатель воспринимается как продолжительность жизни некоторого человека и благодаря этому легко интерпретируется на интуитивном уровне. Как свидетельствуют материалы по национальным проектам «Демография» и «Здравоохранение» этот показатель не требует комментариев, в то время как более простой показатель «коэффициент суммарной рождаемости» (КСР) без комментариев не воспринимается. Однако, на наш взгляд, успешное понимание смысла показателя на интуитивном, бытовом уровне часто мешает правильной интерпретации. О человеке, прожившем 75 лет, можно сказать, что он прожил долгую жизнь, но значение ОПЖ на уровне 75 лет для современной европейской страны оценивается как низкая величина.

Для определения ОПЖ в некоторый период времени необходимо рассчитать таблицу смертности (таблицу дожития) для изучаемого населения. При использовании современной вычислительной техники это совсем не сложно, но трудность в том, что метод расчета таких таблиц до сих пор не унифицирован, не существует одного единственно правильного метода расчета таблицы смертности. Список названий методов расчета, которые использовались после Второй мировой войны, наверняка, займет не одну страницу. В России метод расчета официальных таблиц смертности за этот период менялся не менее 5 раз. Отдел населения ООН, ВОЗ и Евростат пользуются разными методами расчета таблиц. Речь идет о странах, представивших полные данные демографической статистики, необходимые для расчета.

В странах, где анализом смертности занимаются разные исследовательские центры или группы демографов, несовпадение результатов расчета ОПЖ - частое явление. И пусть различия невелики, но они достаточны для того, чтобы с осторожностью относится не только ко второму после запятой знаку в значении ОПЖ, но зачастую и к первому.

Расчет ОПЖ иногда дает противоречащие логике результаты. В конце 1980-х годов, когда начали публиковаться таблицы смертности по республикам СССР, обнаружилось, что если таблицы смертности по всему, городскому и сельскому населению республики рассчитываются по стандартным правилам как для независимых совокупностей, то ОПЖ для всего населения может оказаться больше или меньше, чем для городского и для сельского населения, т.е. не быть некоей средней величиной для двух субпопуляций, что противоречит логике.

Случается, что у надежного в целом метода расчета таблиц смертности существует свой «скелет в шкафу», о котором не любят говорить, но стремятся обойти. Так, в настоящее время самая популярная формула для перехода от возрастного коэффициента смертности к вероятности смерти - это формула Чанга (Chiang 1984). Однако в случае однолетних групп возраста эта формула предполагает, что коэффициент смертности не больше 1, а для 
пятилетнего интервала - не больше 0,2. В реальной статистике однолетний коэффициент вполне может быть больше 1. Обычно такие коэффициенты так или иначе уменьшаются.

Даже если авторы таблиц сообщают, каким методом они рассчитывали таблицу смертности, это не гарантирует полную сопоставимость результатов, полученных «одноименным» методом. Гарантией сопоставимости не является даже использование одного и того же программного обеспечения при одинаковом формате данных, который тоже влияет на результат расчета. Могут использоваться данные по однолетним и пятилетним группам возраста, ряды могут продолжаться за возраст 100 лет, но могут заканчиваться открытой возрастной группой $A$ лет и старше. Хорошо если $A=100$, но в истории России встречались варианты и $A=70$. Тогда определение продолжительности предстоящей жизни в возрасте $A$ становится довольно сложной задачей, допускающей множество решений.

Рассчитывая таблицу смертности с помощью пакета программ MortPak, разработанного в ООН, при разном начале открытого возрастного интервала (пакет такую возможность предоставляет) можно получить разный результат.

Алгоритм расчета таблицы смертности может содержать логические разветвления, программа сама решает, что делать, если возрастной коэффициент смертности больше максимума, допускающего применения формулы Чанга, как продолжить вычисления, если знаменатель для расчета коэффициента смертности равен нулю, и как, если нулю равны и знаменатель, и числитель, и так далее.

В данной статье мы постараемся:

1) убедить читателя не переоценивать точность показателя ОПЖ как измерителя уровня смертности;

2) показать, что ОПЖ не может служить исчерпывающей характеристикой уровня смертности в период, для которого рассчитана;

3) продемонстрировать альтернативные, не менее информативные, но проще вычисляемые меры смертности.

\section{ДАННЫЕ}

Эта статья основана на материалах двух баз данных.

Первая - это Коллекция таблиц смертности человека (Human Life-Table Database) (HLD) (Max Planck Institute... 2021), собранная в основном Институтом демографических исследований Общества Макса Планка в Ростоке (Германия) (The Max Planck Institute for Demographic Research in Rostock). На момент написания этой статьи хранилось 10903 пар таблиц смертности мужчин и женщин, которые начинаются от момента рождения.

Коллекция включает как опубликованные, в том числе официальные таблицы смертности, так и таблицы, присланные их авторами, как правило, научными центрами университетов или известными исследовательскими группами. Многие публикации содержат только часть показателей таблиц смертности. Используются лишь публикации, 
позволяющие однозначно восстановить таблицу. Например, содержащие ряд возрастных вероятностей или коэффициентов смертности или чисел доживающих. Обязательно наличие ряда ожидаемой продолжительности жизни для всех возрастов.

B HLD действует следующая процедура. Каждая таблица смертности полностью пересчитывается на основе выбранного, наиболее информативного ряда. Стандартная таблица смертности дополняется оригинальным рядом продолжительностей предстоящей жизни для всех возрастов. Такая таблица как текстовый файл помещается в базу данных. Кроме того, в базу помещается копия оригинальной таблицы смертности, как правило, в формате PDF.

Вторая база данных - База данных о смертности человека (The Human Mortality Database) (HMD) (University of California ... 2021), являющаяся результатом сотрудничества сотрудников Калифорнийского университета в Беркли (University of California, Berkeley) и Института демографических исследований Общества Макса Планка. База содержит информацию о смертности стран за годы, когда эти данные признаны достоверными. В начале 2021 г. она содержала данные о смертности населения 50 стран, их частей или групп населения за период после 1750 г. Обще число пар \{население, год\} составило 4769. Для краткости мы будем также называть пару \{население, год\} наблюдением. Для каждого наблюдения база содержит среднегодовую численность населения по полу и возрасту, числа умерших по полу, возрасту и по треугольникам Лексиса, возрастные коэффициенты смертности и построенные на основе этих показателей таблицы смертности.

Алгоритмы расчетов, которые используются в HLD и HMD подробно изложены на сайтах баз данных.

\section{О ТОЧНОСТИ ПОКАЗАТЕЛЯ ОПЖ}

Для оценки точности расчета показателя ОПЖ мы сравнили для одних тех же таблиц авторские значения ОПЖ со значениями, рассчитанными нами по единому алгоритму.

Лишь у 3374 (30,95\%) мужских таблиц и 3079 (28,24\%) женских авторская ОПЖ с точностью до двух знаков совпадает с расчетной. На наш взгляд, это очень хороший результат.

С другой стороны оказывается, что для 1643 (15,07\%) мужских таблиц и 2886 (26,47\%) женских абсолютная разница двух ОПЖ превосходит 0,2 года. Многие проблемы связаны с оценкой продолжительности предстоящей жизни в последнем, наиболее старшем возрасте, представленном в таблице. Именно поэтому различия у женщин существеннее, чем у мужчин.

Так или иначе, риск ошибки при сравнениях ОПЖ кажется очень высоким. 


\section{О ЧЕМ ГОВОРЯТ ТАБЛИЦЫ СМЕРТНОСТИ КАЛЕНДАРНОГО ПЕРИОДА}

Чтобы определить ОПЖ для некоторого календарного периода, надо рассчитать некоторым способом таблицу смертности. Таблица представляет собой модель изменения с возрастом численности некоторого поколения людей, которые родились и прожили жизнь под действием смертности изучаемого периода. Это поколение называют гипотетическим или условным. Наверное, появись модель в наши дни, его назвали бы виртуальным поколением. Таблица смертности содержит данные об уменьшении с возрастом численности условного поколения и о том, сколько лет членам поколения предстоит еще прожить в каждом возрасте.

В середине прошлого века официальное название таблиц смертности звучало как «таблицы смертности и средней продолжительности жизни» (ЦСУ СССР 1962). Слово «ожидаемая» заменило слово «средняя» только в конце 1980-х годов.

Развернутое название указывает на то, что определение ОПЖ считалось главной целью расчета таблиц смертности календарного периода. В какой мере верно это предположение?

Для практических целей, таких как демографический прогноз или оценки населения, нужны коэффициенты смертности и легко вычисляемые на их основе вероятности смерти или дожития и вероятности прожить календарный год. Этот последний показатель в России называют коэффициентом дожития и описывают как отношение соседних чисел живущих в условном поколении в возрасте $x+1$ и $x$. Отсюда якобы следует, что область применения чисел живущих не только расчет ОПЖ. Но есть простая формула, которая почему-то не встречается в российских учебниках. Коэффициент дожития в возрасте х равен $P_{x}=\frac{2-M_{x+1}}{2+M_{x}}$, где $M_{x}-$ коэффициент смертности в этом возрасте.

Показатели таблиц смертности участвуют в расчете интегральных характеристик воспроизводства, таких как чистый коэффициент воспроизводства населения, которые в последнее время используют редко.

Показатели таблиц могут быть полезны при сравнении смертности в двух календарных периодах. В частности, ряды показателей применяют для оценки вклада возрастных интервалов в изменение ОПЖ (Андреев 1982). Демографы умеют использовать эти показатели для разных полезных расчетов. Но проблема в том, что все показатели, охватывающие сколько-нибудь продолжительный возрастной интервал, не имеют никакого отношения к реальности. Их польза в том, что они помогают описывать изменения в смертности, так сказать, «широким мазком». Однако, поскольку все возрастные показатели смертности относятся к разным поколениям, результаты вполне могут оказаться неустойчивыми во времени. Анализируя данные о смертности в России в последние годы, мы неоднократно обнаруживали, что вклад отдельных возрастных интервалов в изменение ОПЖ заметно меняется год от года. К тому же такой расчет можно сделать и без таблиц смертности только на основе возрастных коэффициентов. 
Идея условного поколения возникла в XIX веке, когда смертность менялась медленно, и казалось, что нет разницы между условным и реальным поколениями. Внимательно читая книгу С.А. Новосельского (1916), неожиданно понимаешь, что автор сравнивает страны вне времени, период, для которого рассчитана таблица смертности, представляется ему несущественным. В то время никто не стремился различать возрастные изменения смертности в условном и реальном поколениях.

Во второй половине XX века, когда уровень смертности в большинстве развитых стран снижался чрезвычайно быстро, ряды возрастных интенсивностей смертности условных и реальных когорт не утратили сходства, но различия между ними стали очевидны. На рисунке 1 мы сравнили кривую возрастных коэффициентов смертности поколения 1946 г. рождения в Англии и Уэльсе с аналогичными кривыми для трех условных поколений 1951, 1981 и 2011 г. Когортная кривая отражает 2 процесса: изменение смертности с возрастом и снижение со временем. Она как бы протыкает кривые для календарных лет.

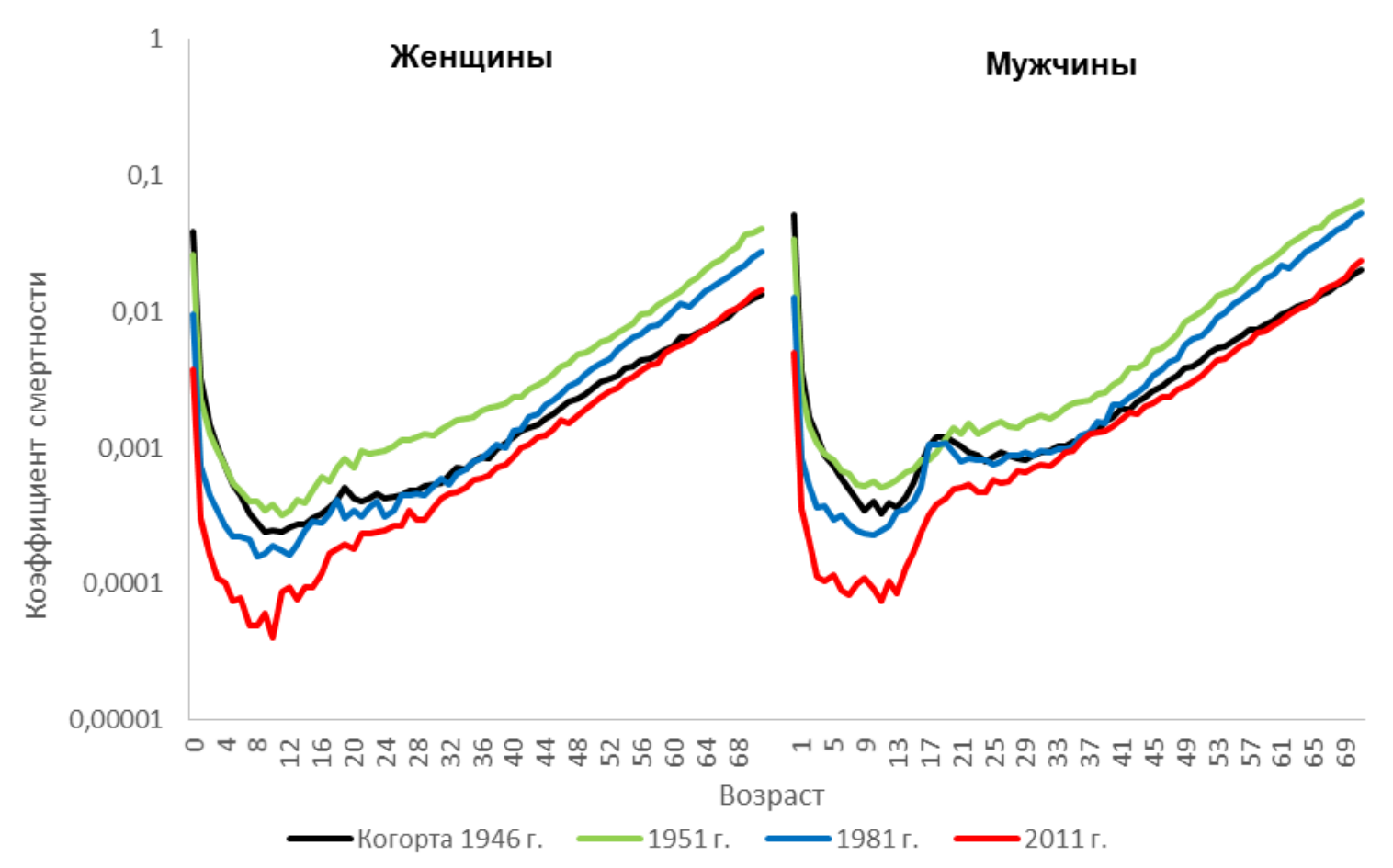

\section{Рисунок 1. Сравнение возрастных кривых смертности когорты 1946 г. и условных поколений 1951, 1981 и 2011 гг., Англия и Уэльс, все население. Полулогарифмическая шкала.}

Источник: (University of California... 2021).

В России достаточно надежные данные существуют только после 1959 г., поэтому кривые на рисунке 2 - приблизительные оценки автора. Поскольку снижение смертности в старших возрастах в России началось много позже, чем в Западной Европе, линии для реального и условного поколений после 50 лет лежат ближе друг к другу. Тем не менее рисунки 1 и 2 показывают, что в обоих случаях едва ли можно использовать таблицы для 
условных поколений с целью оценки шансов дожить среднему человеку до пенсионного возраста.

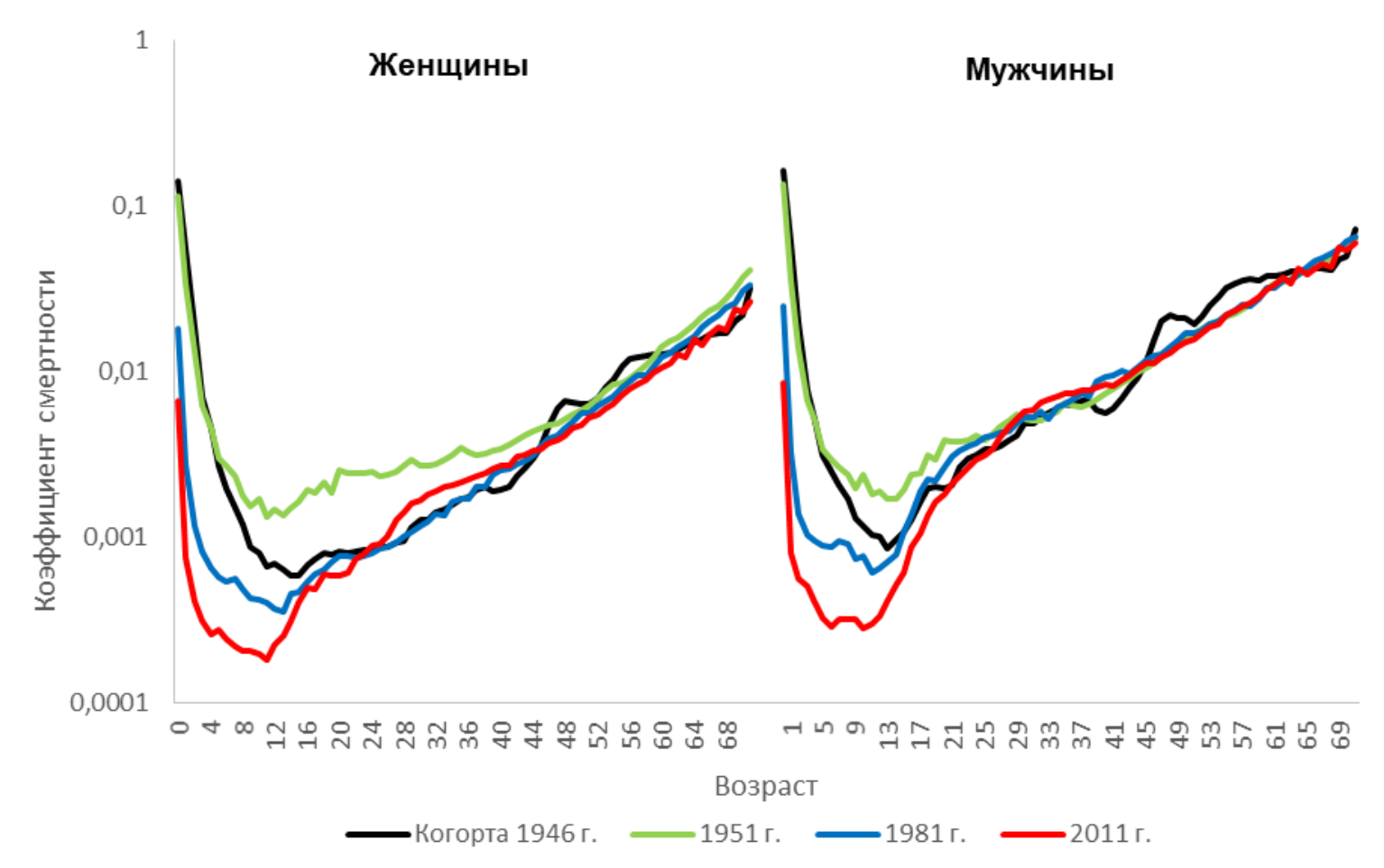

Рисунок 2. Сравнение возрастных кривых смертности когорты 1946 г. и условных поколений 1951, 1981 и 2011 г. Российская Федерация. Полулогарифмическая шкала.

Источник: Рассчитано на основе (Андреев, Дарский, Харькова 1997) и данных (Российская экономическая школа 2021).

Еще одно обоснование полезности расчета таблиц смертности основано на том, что они позволяют судить о закономерностях смертности реальных поколений. Несомненно, кривые показателей для условного поколения похожи на кривые для реального поколения. Но это весьма поверхностное сходство.

Известно, что зависимость интенсивности смертности от возраста хорошо описывается кривой Гомперца. Лет сорок назад отечественная демография пережила попытки объяснить это с помощью моделей старения (Гаврилов, Гаврилова 1979; Шукайло 1979) (см. также (Стрелер 1964)). Повсеместно в объяснениях речь шла о накоплении с возрастом в человеческом организме разного рода неполадок и о том, как это влияет на смертность поколения. Но кривую удавалось подобрать только для показателей условных поколений. В реальных когортах она не работает. Привычка рассчитывать таблицы для условных поколений сделало их реальнее реальных.

Типовые таблицы смертности (Coale, Demeny 1966; United Nations 1982) позволяют думать, что по величине ОПЖ можно предугадать кривую возрастных показателей смертности. Если это действительно верно, то только для условных поколений, для которых типовые таблицы и рассчитывают. 
На рисунке 3 представлены возрастные показатели смертности реальной когорты 1920 г. рождения и условных поколений с той же ОПЖ. Для женщин это условное поколение 1939 г. рождения. ОПЖ в женской когорте 66,91, а в условном - 66,24. Для мужчин взято условное поколение 1932 г., ОПЖ в обоих случаях 59,01.

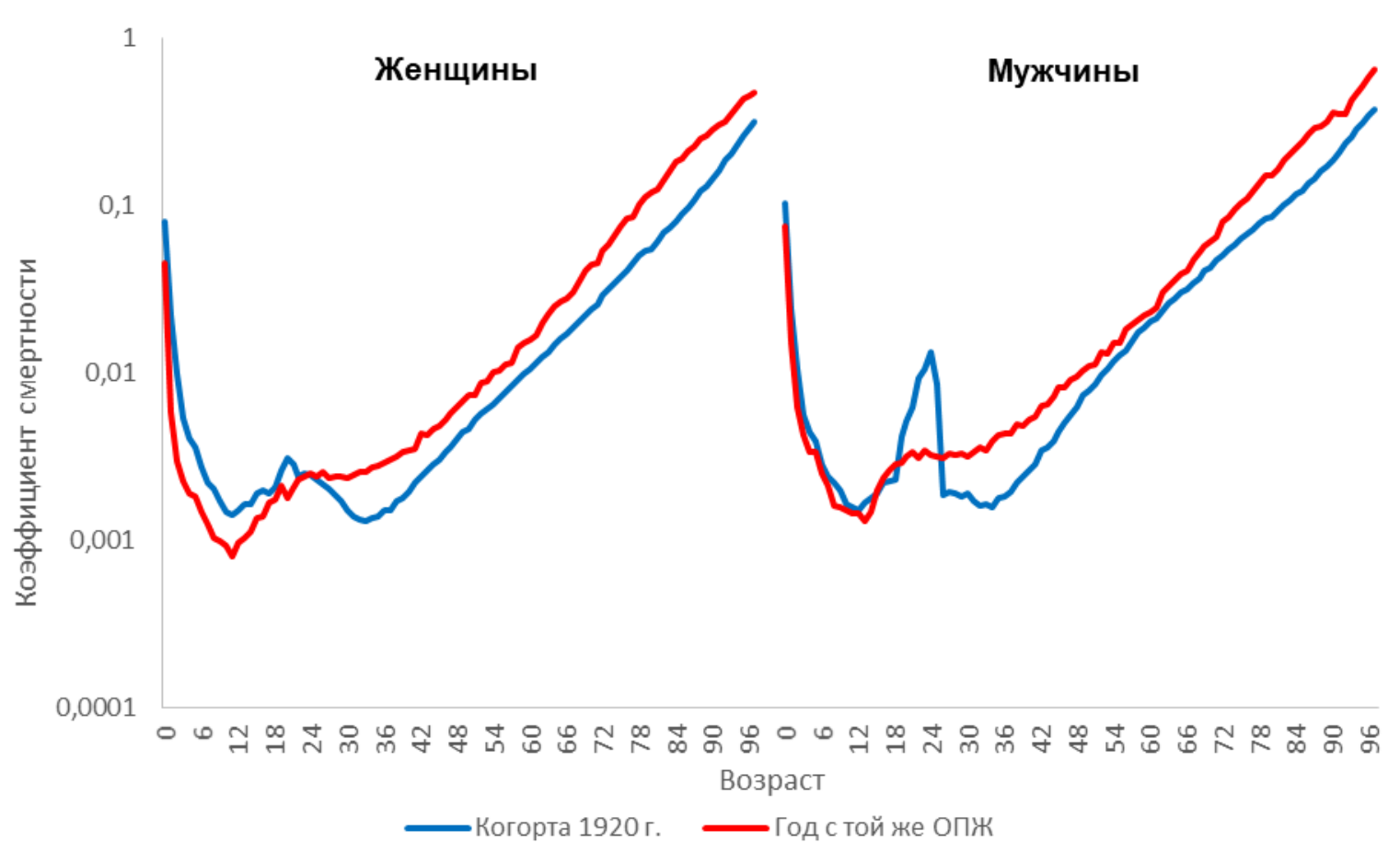

Рисунок 3. Сравнение возрастных кривых смертности когорты 1920 г. и условных поколений с такой же ОПЖ. Англия и Уэльс, все население. Полулогарифмическая шкала.

Источник: (University of California... 2021).

Мы не будем спорить, если читатель сочтет этот пример «не вполне честным», так как поколение 1920 г. пережило войну. К сожалению, для поколений, родившихся после войны, рассчитать ОПЖ невозможно. Конечно, последствия войны нельзя сравнивать с последствиями эпидемии гриппа, летней жары или COVID-19. Но на примере довоенных когорт легче увидеть главные различия таблиц для реальных и условных поколений. В условных поколениях смертность меняется одновременно во всех возрастах, в реальных - на небольшом участке возрастов.

Наш анализ позволяет думать, что главная причина систематического расчета таблиц смертности, который проводят статистические офисы всех развитых стран, международные организации и отдельные исследователи, связана именно с необходимостью определять ОПЖ как интегральной характеристики населения.

\section{АЛЬТЕРНАТИВА ОПЖ}

Обнародованная 27 февраля 1661 г. таблица смертности Дж. Граунта не содержала показателя ОПЖ или аналогичного. Показатель этого типа появился несколько десятилетий 
позже - в работах Х. Гюйгенса, который использовал таблицу Граунта в исследованиях по теории вероятности и ввел понятие математического ожидания (ОПЖ как математическое ожидание события смерти).

Нам не удалось узнать, кто ввел понятие коэффициента смертности как отношения числа смертей за период к числу человеко-лет, прожитых в этот период. Этот показатель принципиально отличается от вероятности смерти, представляющей собой отношение числа смертей к численности на начало временного интервала. Отметим, что Граунт не соотносил число умерших и живых, но рассматривал распределение умерших по возрасту.

Представляется, что к концу XVIII века уже никто не сравнивал числа смертей безотносительно к численности населения. Но, очевидно, общий коэффициент смертности в равной мере зависит от уровня возрастной смертности и от возрастного состава населения. Если исследователя интересует именно смертность, то этот показатель ему не подходит. В середине XIX века У. Фарр разработал метод стандартизации общих коэффициентов смертности. Теперь этот метод в учебниках называют прямым методом стандартизации.

Итак, в середине XIX века появился второй интегральный показатель уровня смертности - стандартизованный коэффициент смертности (СКС). Для этого потребовалось примерно 150 лет с момента начала математического осмысления явления.

Сам по себе общий коэффициент смертности не может рассматриваться как интегральная характеристика уровня смертности, так как в равной мере зависит от возрастных интенсивностей смертности и возрастного состава населения. Он измеряет средний риск смерти в конкретном населении. На наш взгляд, этот показатель не так бесполезен, как кажется. Из него следует, что для того, чтобы снизить риск смерти в «старом» населении, надо заботиться о смертности пожилых, а в «молодом» населении о младенческой смертности.

СКС, как и ОПЖ, можно связать с демографической моделью, но с моделью не поколения, а населения, в котором возрастная структура постоянна и соответствует стандарту, а возрастная смертность такая же, как в исследуемом населении. СКС есть общий коэффициент смертности в этом модельном населении.

Косвенный и обратный методы стандартизации показателей смертности используются в основном в отсутствии полной информации о населении. Косвенный - если неизвестно распределение умерших по возрасту, обратный - в отсутствии сведений о возрастном составе населения. Косвенный метод появился одновременно с прямым, а обратный - гораздо позже, в середине XX века. Для обоих методов требуется определить стандартную функцию возрастной смертности.

При косвенном методе рассчитывается общее число умерших при известном возрастном составе живущих и некоторой теоретической возрастной смертности. Отношение известного общего числа умерших к результату расчета называют стандартизованным индексом смертности.

При обратном методе делением возрастного числа умерших на возрастной коэффициент смертности рассчитывается численность населения, которое могло бы дать 
данный ряд распределенных по возрасту смертей при стандартной возрастной смертности. Отношение реальной общей численности к расчетной также дает индекс смертности.

Прежде чем определить ОПЖ, надо выбрать алгоритм расчета таблицы смертности, основанном на том или преобразовании возрастных коэффициентов смертности. Алгоритм стандартизации общего коэффициента смертности определяется стандартным населением. Можно выбрать один из общеизвестных стандартов, можно использовать свой стандарт. Чтобы публикация не вызвала недовольство читателя, достаточно этот стандарт опубликовать.

В настоящее время наиболее часто используется Европейский стандарт ВОЗ 1976 г. и стандарт Евростата 2013 г. (Eurostat 2013). Стандарт 1976 г, безусловно, устарел, так как предусматривает открытый возрастной интервал 85 лет и старше, тогда как снижение смертности после 85 лет становится все более важным фактором снижения смертности.

В отличие от ОПЖ, СКС аддитивен по причинам смерти, поэтому несложно оценить вклад отдельных причин смерти в его изменение. Несколько сложнее оценивать вклад отдельных возрастных групп, так как придется учитывать их доли в стандартном населении. Но ничего похожего на метод декомпозиции различий в ОПЖ (Андреев 1982) не потребуется.

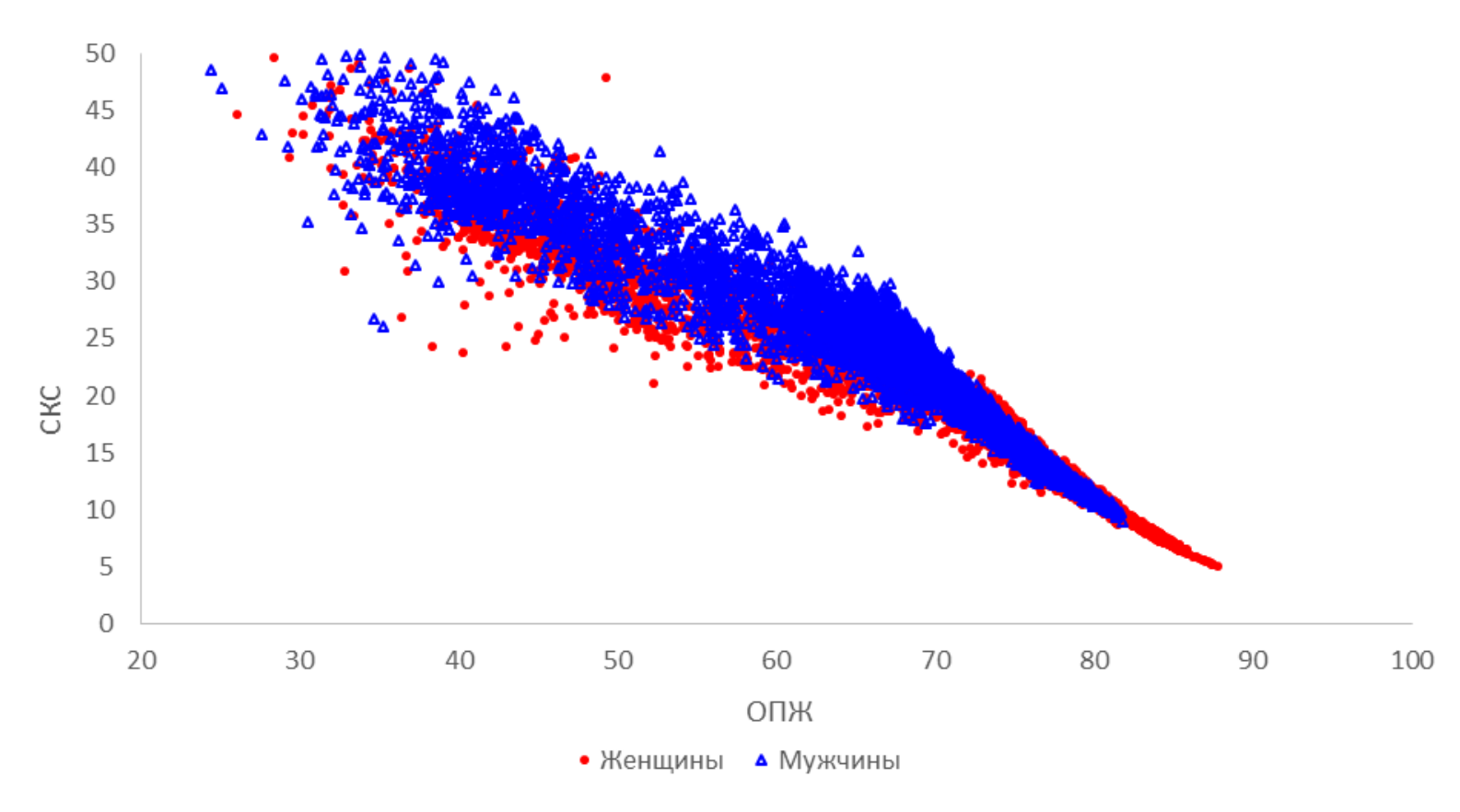

Рисунок 4. Соотношение стандартизованного коэффициента смертности на основе Европейского стандарта 2013г. и ОПЖ мужчин и женщин по данным НMD (University of California 2021) (4769 наблюдений)

Источник: Расчеты автора.

К сожалению, индексы смертности, рассчитанные и косвенным и обратным методом, этими свойствами не обладают. Нельзя, зная индексы смертности от отдельных причин, оценивать их вклад в общий индекс. 
Мы воспользовались данными HMD, чтобы попытаться сравнить 2 интегральных показателя. Нас интересует вопрос, в какой мере согласуются полученные с их помощью оценки уровня смертности.

На рисунке 4 представлено соотношение СКС и ОПЖ во всех парах \{население, год\} для мужчин и для женщин. Было использовано Европейское стандартное население 2013 г.

Рисунок показывает, что связь, видимо, не зависит от пола и в подавляющем числе случаев четко прослеживается. Эта связь заведомо нелинейная, что особенно четко проявляется при высоких ОПЖ и низких СКС, так как нулевому СКС соответствовала бы бесконечная продолжительность жизни.

Связь между СКС и ОПЖ все же достаточно четкая, чтобы для каждого значения одного показателя можно было посчитать среднее значение другого. По нашим расчетам, ОПЖ 78 лет, которую Россия, согласно национальной доктрине, стремится достигнуть в 2030 г., соответствует в среднем СКС, равному 12,4 на 1000 (если взять Европейское стандартное население 2013 г.), при диапазоне значений от 11,4 до 13, 3.

Для оценки согласованности различных характеристик смертности полезно воспользоваться непараметрическим коэффициентом ранговой корреляции tau_b Кенделла, основанном на подсчете совпадении рангов (таблица 1).

В нашем случае идеально было бы, если ранги шли в обратном порядке. Расчет с включением данных для мужчин и женщин показал, что коэффициент составляет $-0,89$, что для коэффициента Кенделла очень высокое по абсолютной величине значение. Аналогичный расчет на показателях для двух полов вместе дал практически тот же результат $-0,90$ (таблица 2).

Таблица 1. Коэффициент ранговой корреляции tau_b Кенделла по массиву 9538 наблюдений, мужчины или женщины

\begin{tabular}{r|lr|r|r|r|r|r}
\hline & & \multicolumn{1}{c|}{1} & \multicolumn{1}{c|}{2} & \multicolumn{1}{c|}{3} & \multicolumn{1}{c}{5} & \multicolumn{1}{c}{5} & \multicolumn{1}{c}{6} \\
\hline 1 & ОПЖ & 1 & $-0,941$ & $-0,887$ & $-0,837$ & $-0,876$ & $-0,622$ \\
2 & СКС, стандарт HМD-21 & $-0,941$ & 1 & 0,944 & 0,826 & 0,840 & 0,633 \\
3 & СКС, Европейский стандарт 2013 & $-0,887$ & 0,944 & 1 & 0,798 & 0,799 & 0,625 \\
4 & Индекс смертности косвенным методом & $-0,837$ & 0,826 & 0,798 & 1 & 0,893 & 0,673 \\
5 & Индекс смертности обратным методом & $-0,876$ & 0,840 & 0,799 & 0,893 & 1 & 0,641 \\
6 & Общий коэффициент смертности & $-0,622$ & 0,633 & 0,625 & 0,673 & 0,641 & 1 \\
\hline
\end{tabular}

Примечание. Коррелячия значима при уровне ниже 0,01.

Таблица 2. Коэффициент ранговой корреляции tau_b Кенделла для двух полов вместе по 4769 наблюдений

\begin{tabular}{rl|r|r|r|r|r|r}
\hline & & 1 & \multicolumn{1}{c|}{2} & \multicolumn{1}{c}{3} & \multicolumn{1}{c}{4} & \multicolumn{1}{c}{5} & \multicolumn{1}{c}{6} \\
\hline 1 & ОПЖ & 1 & $-0,949$ & $-0,900$ & $-0,913$ & $-0,905$ & $-0,625$ \\
2 & СКС, стандарт HMD-21 & $-0,949$ & 1 & 0,950 & 0,929 & 0,881 & 0,634 \\
3 & СКС, Европейский стандарт 2013 & $-0,900$ & 0,950 & 1 & 0,908 & 0,843 & 0,628 \\
4 & Индекс смертности косвенным методом & $-0,913$ & 0,929 & 0,908 & 1 & 0,897 & 0,696 \\
5 & Индекс смертности обратным методом & $-0,905$ & 0,881 & 0,843 & 0,897 & 1 & 0,661 \\
6 & Общий коэффициент смертности & $-0,625$ & 0,634 & 0,628 & 0,696 & 0,661 & 1 \\
\hline
\end{tabular}

Примечание. Коррелячия значима при уровне ниже 0,01. 
Хотя распределения наблюдений, основанные на СКС и на ОПЖ, дают очень близкие представления о том, что демографы воспринимают как «уровень смертности», между двумя способами классификации наблюдений есть принципиальное отличие. ОПЖ как всякий показатель продолжительности жизни чутко реагирует на возраст умершего. Чем моложе умерший, тем больше его смерть снижает ОПЖ. Мы не уверены, что это свойство полезно показателю, характеризующему уровень смертности населения в некотором году. Реакция на возраст СКС зависит от выбранного стандартного населения. Европейский стандарт 2013 г. мало различает возраста моложе 65 лет.

Известно, что в конце XIX века Европейская Россия отличалась ужасающе высокой младенческой смертностью. Согласно таблицам смертности населения Европейской России в границах РСФСР в конце 1920-х годов, в 1896-97 гг. до 1 года не доживали 303 из 1000 новорожденных. В значительной мере именно поэтому ОПЖ составляла 30,5 года (Смертность и продолжительность жизни... 1930). По нашим расчетам на основе этих таблиц смертности СКС составлял 36,0 на 1000. Мы обнаружили в HMD 272 наблюдения с СКС от 35 до 37 на 1000. Средний СКС в этой совокупности равен российскому, а ОПЖ 43,3 года. Такая величина СКС зафиксирована во Франции в 1944 г. (все национальное население). При этом ОПЖ во Франции для того же года составляла 55,8 года. Данное соотношение ОПЖ и СКС вызвано тем, что СКС лучше уловил высокую смертность взрослого населения. Таким образом, ОПЖ как мера уровня смертности населения как бы переоценивает роль смертности детей и недоценивает роль смертности взрослых. Однако эта проблема заслуживает специального рассмотрения.

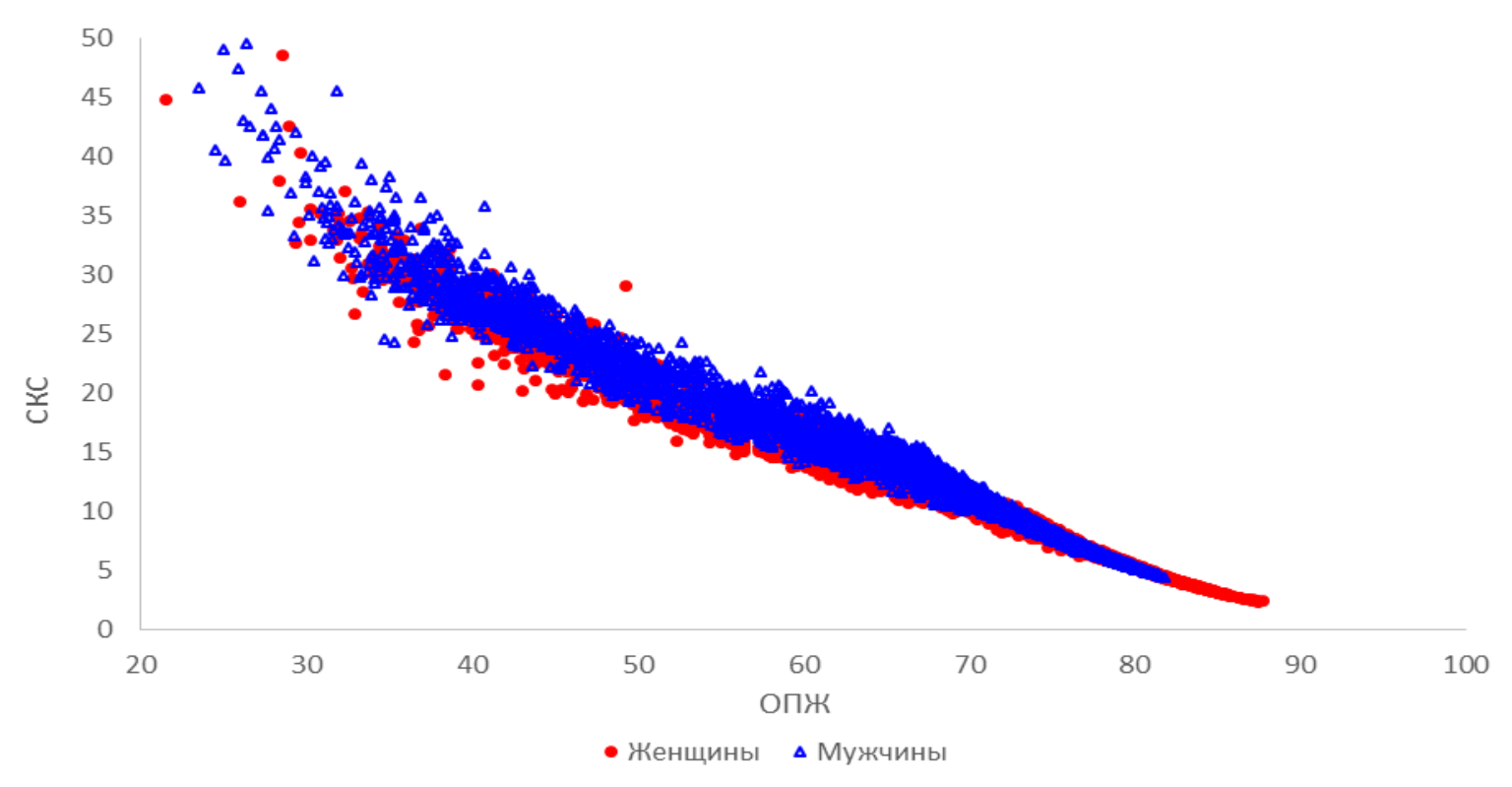

Рисунок 5. Соотношение стандартизованного коэффициента смертности с использованием средней для НMD возрастной структуры на начало 2021 г. (HMD-21) и ОПЖ мужчин и женщин по данным HMD (4769 наблюдений)

Источник: Расчеты автора.

Нельзя забывать, что Евростат, разрабатывая стандартное население, ориентировался на структуры населения развитых стран Европы. 
Немонотонность возрастной структуры наводит на мысль, что его предполагалось через 5-10 лет пересмотреть. Так или иначе, стандарт не предполагалась применять для столь широкого списка стран. Мы решили взять в качестве стандарта среднюю возрастную структуру для двух полов вместе по всему списку из 4769 населений и лет. Чтобы как-то отличать этот стандарт, мы назвали его HMD-21. Стандарт HMD-21 представлен в таблице П1 Приложения.

При использовании стандарта HMD-21 коэффициент ранговой корреляции Кенделла двух показателей - ОПЖ и СКС - равен -0,94 при объединении показателей, определенных независимо для мужчин и женщин, в один массив данных (таблица 1) и -0,95 при расчете показателей для двух полов взятых вместе (таблица 2).

Рисунок 5 свидетельствует, что при использовании средней возрастной структуры HMD-21 в качестве стандарта, связь между ОПЖ и СКС выглядит четче, чем при использовании Европейского стандарта населения 2013 г. (ср. рис. 4 и 5).

Для расчета индексов смертности косвенным и обратным методом мы рассчитали стандартный ряд возрастных коэффициентов смертности для двух полов вместе как среднюю по всем населениям-годам. Использован краткий ряд возрастных показателей HMD с открытым интервалом 95 и более лет (таблица П2 Приложения). Коэффициент Кенделла для индексов показывает их весьма высокую согласованность с ОПЖ, хотя и более низкую, чем у обоих СКС.

\section{ПРИБЛИЖЕННЫЕ ОЦЕНКИ}

Низкие по сравнению с другими показатели в последних строке и столбце таблиц 1 и 2 еще раз убеждают, что общий коэффициент смертности не может служить интегральной характеристикой уровня смертности вместо ОПЖ. Но все же показатели корреляции tau_b Кенделла довольно высоки. Отсюда возникла идея использовать общий коэффициент при приближенных оценках.

Возрастная структура населения при пятилетних группах возраста, как правило, меняется довольно медленно. Поэтому можно допустить, что на коротком временном интервале изменения СКС пропорциональны изменениям общего коэффициента смертности. Мы попытались это проверить.

В данном случае в качестве стандарта для расчета CKC мы взяли стандарт HMD-21. Среди данных HMD мы выбрали все такие случаи, когда сведения о некотором населении имеются за два последовательных года. Таких случаев оказалось 4718. Мы вычислили СКС для вторых из двух лет, исходя из допущения, что изменения СКС пропорциональны изменениям общего коэффициента, а затем сравнили оценку с «правильным» показателем и рассчитали относительную ошибку оценки. Результат обнадеживает: в 90\% всех случаев ошибка лежит в интервале $\pm 3 \%$. Это верно и для мужчин, и для женщин, и для двух полов вместе. 
Можно ожидать, что вероятность не ошибиться высока, но это не всегда так. Ошибки выше 10\% или ниже -10\% случаются для периода до 1920 г., а также для Исландии или Новой Зеландии (только маори).

Напротив, наши попытки судить об изменении ОПЖ только на основе изменения общего коэффициента смертности или общего числа умерших успехом не увенчались. Коэффициент ранговой корреляции между изменениями ОПЖ и общего коэффициента при том же числе наблюдений составил $-0,77$ (уровень значимости 0,01). Весьма высоким по абсолютной величине оказался и коэффициент корреляции Пирсона $(-0,91)$. Дальнейший анализ показал, что возможна только линейная модель. Но точность модели совершенно не удовлетворительна. Среднее абсолютное отклонение предсказанной ОПЖ от факта равно 0,34 года, в трети всех наблюдений отклонение больше четверти года.

\section{НЕКОТОРЫЕ ИТОГИ}

Чтобы узнать ОПЖ в населении в некоторый период, необходимо рассчитать таблицу смертности для условного поколения этого периода. Результат расчета зависит от формата доступных данных о численности населения по возрастам и о возрастных числах умерших, а также от избранного алгоритма расчета. Формат данных определяется состоянием статистики населения и позицией национальной статистической службы. Чем детальнее данные, тем заметнее их дефекты. Избегая возможной критики, статистические офисы нередко публикуют только агрегированные данные.

Метод расчета зависит как от доступных данных, так и от субъективных предпочтений и знаний авторов расчета. В России, например, до сих пор популярен метод американского статистика Гревилла (Greville 1943), но мало кто знаком с методами, которые используются в Центрах по контролю и профилактике заболеваний (Centers for Disease Control and Prevention) США в современных таблицах (Anderson 1999).

С помощью Коллекции таблиц смертности человека мы продемонстрировали, что десятичные знаки в величине ОПЖ существенно зависят от метода расчета. Таким образом, ОПЖ не является самой точной характеристикой уровня смертности.

Мы показали, что условные поколения XX-XXI веков мало похожи на реальные поколения. Поэтому делать какие-то выводы о реальных поколениях, опираясь на таблицы смертности календарного периода, нельзя. Представляется, что гораздо проще предсказать продолжительность жизни молодых когорт (Shkolnikov et al. 2014), чем продолжительность предстоящей жизни когорт, чьи детство и молодость прошли в 1940-1950-х годах. Известно, что смертность от многих болезней в пожилых возрастах зависит от условий жизни в детстве и юношестве. Невозможно предугадать суммарный эффект этой связи, но ссылки на данные условных поколений вряд ли обоснованы.

Наш вывод: получение величины ОПЖ - главная, а возможно единственная цель расчета таблиц смертности условного поколения. 
Связь между ОПЖ и возрастными коэффициентами смертности описывается очень не простыми соотношениями, поэтому довольно трудно сказать, как те или иные изменения возрастной смертности в целом и от отдельных причин смерти отражаются на ОПЖ.

В сознании непрофессионалов показатель ОПЖ воспринимается как длительность жизни конкретных людей. На нее ссылаются, когда предлагают увеличить пенсионный возраст или, наоборот, доказывают неправомерность такого шага. Подсчет численности людей в условном поколении, которые доживают или не доживают до некоторого возраста, не имеет отношения ни к какой реальной совокупности.

В начале 1970-х годов руководство СССР пришло к выводу, что продолжительность жизни в стране не должна опускаться ниже 70 лет (Андреев 2011), и этот показатель повторялся в международных статистических публикациях. Начиная с 1973 г. в издаваемых ООН Демографических ежегодниках публиковалась продолжительность жизни в 1971-1972 гг. для СССР для мужчин 64 года, для женщин - 74 года, для Украины соответственно 68 и 76, для Белоруссии - 67 и 74. Только для Белоруссии приводилась продолжительность жизни для двух полов, равная 71 году. Лишь в 1989 г. Госкомстат СССР опубликовал фактические таблицы смертности за период с 1970 по 1987 г. В начале 2010-х ОПЖ в России перевалила за 70 лет, и это снова показалось большим достижением.

Если стандартное население выбрано и структура обнародована, а возрастные коэффициенты смертности рассчитаны, то никакие силы не смогут повлиять на значение СКС. Расчет возрастных коэффициентов смертности содержит некоторую неопределенность. Есть разные возможности распределять по возрастам умерших неизвестного возраста, есть несколько способов расчета населения под риском, но все эти неопределенности возникают и при расчете ОПЖ.

СКС - линейная функция возрастных коэффициентов смертности. Поэтому чрезвычайно просто оценить влияние на изменение СКС отдельных причин смерти. Немного сложнее выглядит формула, позволяющая оценить вклад в изменение СКС отдельных возрастных групп. Более сложное выражение позволяет описать вклад в изменение СКС для страны в целом уровня смертности в отдельных ее частях или социальных группах и др. Например, можно сделать рутинной процедурой анализ вклада регионов в динамику смертности в России с использованием СКС, подобный представленному в нашей совместной работе (Timonin et al. 2017).

Можно рассчитывать СКС не для всей шкалы возрастов, а для отдельных интервалов, например для взрослых, рабочих, пенсионных возрастов.

Что касается возможности сравнивать с другими населениями, с другими периодами и так далее, то международные базы данных позволяют рассчитать абсолютно сопоставимые СКС. Кроме HMD, назовем Базу данных о смертности человека по причинам смерти (The Human Cause-of-Death Database) (Institute for Demographic ... 2021) и Базу данных ВОЗ о смертности (World Health Organization 2021). 
Мы не сможем рассчитать СКС для стран, где отсутствует адекватная демографическая статистика, а публикуемые показатели ОПЖ есть результат разного рода косвенных оценок, но, на наш взгляд, это к лучшему.

Используя СКС, мы освобождаемся от содержащегося в определении условного поколения мистического «на протяжении всей жизни». Для объяснения, что такое СКС, можно сказать, что это число умерших на 1000 (или 100 тыс.) человек населения в исследуемом населении, если бы у него была такая же возрастная структура, как в стандартном населении или, наоборот, число умерших в стандартном населении, если бы в нем была такая же смертность, как исследуемом населении.

Конечно, отказаться в одночасье от использования показателя ожидаемой продолжительности жизни для календарного периода не удастся. Но использовать только СКС, проводя анализ динамики смертности или подготавливая статьи, рассчитанные на читателя-демографа, вполне реально.

Когда статья была готова, новейшие статистические публикации заставили сделать еще одно короткое добавление. Предварительные итоги 2020 г. в странах Европы и России свидетельствуют, что ОПЖ - очень мягкий показатель. Так, в России число умерших и ОКС в 2020 г. в условиях пандемии COVID-19 выросли по сравнению с 2019 г. более чем на 18\%, а ОПЖ снизилась по предварительным оценкам на 1,8 года или только на 2,5\% (Росстат 2021a, b, c).

\section{ЛИТЕРАТУРА}

Андреев Е.М. (1982). Метод компонент в анализе продолжительности жизни. Вестник статистики, (9), 42-47.

Андреев Е.М. (2011). Ожидаемая продолжительность жизни 70 лет, или déjà vu отечественной демографии. Демоскоп Weekly, 487- 488.

Андреев Е.М., Дарский Л.Е., Харькова Т.Л. (1997). Демографическая история России: 1927-1959. М.: Информатика.

Гаврилов Л.А., Гаврилова Н.С. (1979). Исследование кинетических закономерностей смертности людей в историческом аспекте. ДАН СССР, 245(4), 1017 - 1020.

Новосельский С.А. (1916). Смертность и продолжительность жизни в России. Петроград: Типография Министерства Внутренних Дел.

Смертность и продолжительность жизни населения СССР 1926-1927 22. (1930). Таблицы смертности. М.; Л.: Планхозгиз.

Стрелер Б. (1964). Время, клетки и старение. Пер. с англ. М.

Российская экономическая школа (2021). Российская база данных по рождаемости и смертности. URL: http://demogr.nes.ru/index.php/ru/demogr_indicat/data (данные загружены 05.02.2021).

Росстат (2021a). Естественное движение населения в разрезе субъектов Российской Федерации по месящам. 2020 год. URL: https://rosstat.gov.ru/folder/12781 (данные загружены 08.02.2021). 
Росстат (2021b). Оценка численности постоянного населения на 1 января 2021 г. и в среднем за 2020 г. URL: https://rosstat.gov.ru/folder/12781 (данные загружены 19.03.2021).

Росстат (2021c). Предварительная оценка ожидаемой продолжительности жизни при рождении по субъектам Российской Федераџии за 2020 год. URL: https://rosstat.gov.ru/folder/12781 (данные загружены 19.03.2021).

ЦСУ СССР (1962). Таблиџы смертности и средней продолжительности жизни населения СССР. 1958-59 г2. М.: Госстатиздат.

Шукайло В.Ф. (1979). О принципах матем. отображения сущности процессов смертности. В Продолжительность жизни: анализ и моделирование (сс. 104-123). М.,

Anderson R.N. (1999). Method for constructing complete nnual U.S. life tables. In: Vital and health statistics. Series 2, Data evaluation and methods research; no. 129.

Chiang C.L. (1984). The Life Table and its Applications. Malabar: Florida.

Coale A., Demeny P. (1966). Regional Model Life Tables and Stable Populations. Princeton: Princeton University Press.

Eurostat (2013). Revision of the European Standard Population - Report of Eurostat's task force edition.

Greville T. (1943). Some methods of constructing abridged life tables, Records of the. American Institute of Actuaries, 32, 29-43.

Institute for Demographic Studies (INED), Max Planck Institute for Demographic Research (2021). The Human Cause-of-Death Database (HCD). URL: https://www.causesofdeath.org/cgi-bin/main.php.

Max Planck Institute for Demographic Research (2021). Human Life Table Database. Retrieved from https://www.lifetable.de/cgi-bin/data.php (data downloaded on 05.02.2021).

Shkolnikov V.M., Jdanov D.A., Andreev E. M., Vaupel J. W. (2014). Rapid increase in maximal country specific life expectancy across cohorts. Advances in Gerontology, 27(2), 229-235.

Timonin S., Danilova I., Andreev E. M., Shkolnikov V. (2017). Recent mortality improvement in Russia: are regions following the same tempo? European Journal of Population, 33(5), 733-763.

United Nations (1982). Model Life Tables for Developing Countries (United Nations publication, Sales No. E.81.XIII.7).

University of California, Berkeley, and Max Planck Institute for Demographic Research (2021). The Human Mortality Database. U.K., United Kingdom Total Population. Retrieved from https://www.mortality.org/cgi-bin/hmd/country.php?cntr=GBR_NP\&level=1 (data downloaded on 05.02.2021) .

World Health Organization (2021). WHO Mortality Database. URL: https://www.who.int/data/data-collection-tools/who-mortality-database. 


\section{ПРИЛОЖЕНИЕ}

Таблица П1. Средняя возрастная структура двух полов вместе населения под риском по всем содержащимся в Human Mortality Database на начало 2021 г. 4769 наблюдениям

\begin{tabular}{|c|c|c|c|c|c|c|c|}
\hline Возраст & 0 & $1-4$ & $5-9$ & $10-14$ & $15-19$ & $20-24$ & $25-29$ \\
\hline Доля & 181757 & 687900 & 834378 & 819342 & 799294 & 771576 & 744360 \\
\hline Возраст & $30-34$ & $35-39$ & $40-44$ & $45-49$ & $50-54$ & $55-59$ & $60-64$ \\
\hline Доля & 714884 & 681483 & 643165 & 600511 & 552383 & 495744 & 433179 \\
\hline Возраст & $65-69$ & $70-74$ & $75-79$ & $80-84$ & $85-89$ & $90-94$ & $95-99$ \\
\hline Доля & 362419 & 283185 & 198846 & 119115 & 54967 & 17649 & 3465 \\
\hline Возраст & $100-104$ & 105-109 & $110+$ & Всего & & & \\
\hline Доля & 373 & 23 & 2 & 10000000 & & & \\
\hline
\end{tabular}

Примечание: Дата загрузки: 05.02.2021.

Таблица П2. Средние возрастные коэффициенты смертности на 1 млн для двух полов вместе по всем содержащимся в Human Mortality Database на начало 2021 г. 4769 наблюдениям

\begin{tabular}{l|c|c|c|c|c|c|c}
\hline Возраст & 0 & $1-4$ & $5-9$ & $10-14$ & $15-19$ & $20-24$ & $25-29$ \\
\hline Коэффициент & 59441 & 8126 & 2225 & 1417 & 2171 & \multicolumn{2}{c|}{3064} \\
\hline Возраст & $30-34$ & $35-39$ & $40-44$ & $45-49$ & $50-54$ & $55-59$ & $60-64$ \\
\hline Коэффициент & 3528 & 4191 & 5275 & 6935 & 9773 & 13935 & 21115 \\
\hline Возраст & $65-69$ & $70-74$ & $75-79$ & $80-84$ & $85-89$ & $90-94$ & $95+$ \\
\hline Коэффициент & 31939 & 50400 & 79487 & 124212 & 190442 & 276548 & 405034 \\
\hline
\end{tabular}

Примечание: Дата загрузки: 05.02.2021. 


\title{
IS LIFE EXPECTANCY AT BIRTH REALLY \\ THE BEST MEASURE OF MORTALITY IN A POPULATION?
}

\section{EVGENY ANDREEV}

\begin{abstract}
It is generally considered that the most adequate indicator of mortality in any population in a certain calendar period is life expectancy at birth (LE). Yet this indicator has serious drawbacks, and its use leads to a number of unjustified difficulties. The main limitation is that the method for calculating life expectancy is still not unified and most likely never can be. As a result, LE for different countries and periods may not be comparable. The data of the international Human Life Table Database makes it possible to demonstrate that in LE comparisons it is often impossible to use the first decimal digit.
\end{abstract}

Computation of LE requires the calculation of life tables for a hypothetical cohort corresponding to the calendar period under consideration. When mortality declines rapidly, as has been the case in most countries since the mid-20th century, life tables for a hypothetical cohort say little about the age patterns of birth cohort mortality, and instead form false impressions. Counting how many people in a hypothetical cohort survived or did not survive to a certain age has no relation to any real population.

The transition from age-specific mortality rates to life expectancy is described not by a formula but by a computational procedure. This creates serious difficulties when trying to assess the impact of mortality at certain ages from certain causes of death in certain regions and population groups.

All these drawbacks are not shared by the standardized death rate (SDR), the calculation of which becomes the only option as soon as the standard population is specified. Data of the Human Mortality Database allowed us to show that mortality level estimations based on SDR almost coincide with those based on LE.

SDR is a linear function of age-specific mortality rates; therefore, it is not difficult to calculate the effect of mortality in certain ages, regions or population groups and from different causes of death on SDR.

Non-professionals perceive LE on an intuitive level as the duration of a human life, which is rather a drawback of the indicator. The use of SDR requires more explanation. But in practical research and in professional publications, focusing on SDR will facilitate the work and protect against unwarranted emotions.

Key words: mortality, life expectancy, standardized mortality rate, mortality rate.

EvgENy ANDREev (e.andreev@hse.ru), NATIONAl RESEARCh University Higher School of Economics, RusSia.

DATE RECEIVED : MARCH 2021.

\section{REFERENCES}

Anderson R.N. (1999). Method for constructing complete nnual U.S. life tables. In Vital and health statistics, Series 2, Data evaluation and methods research, no. 129.

Andreev E.M., Darskiy L.E., Kharkova T.L. (1997). Demograficheskaya istoriya Rossii: 19271959 [Demographic History of Russia: 1927-1959]. Moscow: Informatika.

Andreyev E.M. (1982). Metod komponent v analize prodolzhitel'nosti zhizni [Component method in life expectancy analysis]. Vestnik statistiki, (9), 42-47.

Andreyev E.M. (2011). Ozhidayemaya prodolzhitel'nost' zhizni 70 let, ili déjà vu otechestvennoy demografii [Life expectancy of 70 years, or déjà vu of domestic demography]. Demoskope Weekly, 487- 488. 
Chiang C.L. (1984). The Life Table and its Applications. Malabar: Florida.

Coale A., Demeny P. (1966). Regional Model Life Tables and Stable Populations. Princeton: Princeton University Press.

Eurostat (2013). Revision of the European Standard Population - Report of Eurostat's task force edition.

Gavrilov L.A., Gavrilova N.S. (1979). Issledovaniye kineticheskikh zakonomernostey smertnosti lyudey v istoricheskom aspekte [Life expectancy of 70 years, or déjà vu of domestic demography]. DAN SSSR, 245(4), 1017-1020.

Greville T. (1943). Some methods of constructing abridged life tables, Records of the. American Institute of Actuaries, 32, 29-43.

Institute for Demographic Studies (INED), Max Planck Institute for Demographic Research (2021). The Human Cause-of-Death Database (HCD). URL: https://www.causesofdeath.org/cgi-bin/main.php

Max Planck Institute for Demographic Research (2021). Human Life Table Database. Retrieved from https://www.lifetable.de/cgi-bin/data.php (data downloaded on 05.02.2021).

New Economic School (2021). The Russian Fertility and Mortality database. Retrieved from http://www.demogr.nes.ru/index.php/en/demogr_indicat/data (data downloaded on 05.02.2021).

Novosel'skiy S.A. (1916). Smertnost' i prodolzhitel'nost' zhizni v Rossii [Mortality and Life Expectancy in Russia]. Petrograd: Tipografiya Ministerstva Vnutrennikh Del.

Rosstat (2021a). Yestestvennoye dvizheniye naseleniya v razreze sub"yektov Rossiyskoy Federatsii po mesyatsam. 2020 god. [Vital statistics data on regiohs of the Russian Federation by months. 2020 year]. URL: https://rosstat.gov.ru/folder/12781 (data downloaded on 08.02.02.2021).

Rosstat (2021b). Otsenka chislennosti postoyannogo naseleniya na 1 yanvarya 2021 g. iv srednem za $2020 \mathrm{~g}$. [Estimated resident population as of January 1, 2021 and on average for 2020]. URL: https://rosstat.gov.ru/folder/12781 (data downloaded on 19.03.2021).

Rosstat (2021c). Predvaritel'naya otsenka ozhidayemoy prodolzhitel'nosti zhizni pri rozhdenii po sub"yektam Rossiyskoy Federatsii za 2020 god [Preliminary estimate of life expectancy at birth for the subjects of the Russian Federation for 2020]. URL: https://rosstat.gov.ru/folder/12781 (data downloaded on 19.03.2021).

Shkolnikov V.M., Jdanov D.A., Andreev E. M., Vaupel J.W. (2014). Rapid increase in maximal country specific life expectancy across cohorts. Advances in Gerontology, 27(2), 229-235.

Shukaylo V.F. (1979). O printsipakh matem. otobrazheniya sushchnosti protsessov smertnosti [On the principles of mat. displaying the essence of mortality processes]. In Prodolzhitel'nost' zhizni: analiz i modelirovaniye (pp. 104-123). Moscow.

Smertnost' i prodolzhitel'nost' zhizni naseleniia SSSR 1926-1927 gg. [Mortality and life expectancy of the USSR 1926-1927] (1930). Tablitsy smertnosti. Moscow - Leningrad.

Strehler B. (1964). Vremya, kletki i stareniye [Time, cells, and aging]. Moscow.

Timonin S., Danilova I., Andreev E. M., Shkolnikov V. (2017). Recent mortality improvement in Russia: are regions following the same tempo? European Journal of Population, 33(5), 733-763. 
TSSU SSSR (1962). Tablitsy smertnosti i sredney prodolzhitel'nosti zhizni naseleniya SSSR. 1958-59 [Life tables and life expectancy of the population of the USSR. 1958-59]. Moscow: Gosstatizdat.

United Nations (1982). Model Life Tables for Developing Countries (United Nations publication, Sales No. E.81.XIII.7).

University of California, Berkeley, and Max Planck Institute for Demographic Research (2021). The Human Mortality Database. U.K., United Kingdom Total Population. Retrieved from https://www.mortality.org/cgi-bin/hmd/country.php?cntr=GBR_NP\&level=1 (data downloaded on 05.02.2021).

World Health Organization (2021). WHO Mortality Database. URL: https://www.who.int/data/data-collection-tools/who-mortality-database 